\title{
Behavioral decline and premature lethality upon pan-neuronal ferritin overexpression in Drosophila infected with a virulent form of Wolbachia
}

\section{Stylianos Kosmidis, ${ }^{1,2}$, Fanis Missirlis ${ }^{3,4}{ }^{*}$, Jose A. Botella ${ }^{5}$, Stephan Schneuwly ${ }^{5}$, Tracey A. Rouault ${ }^{3}$ and Efthimios M. C. Skoulakis ${ }^{1}$}

${ }^{1}$ Neuroscience Division, Biomedical Sciences Research Centre "Alexander Fleming," Vari, Greece

${ }^{2}$ Department of Neuroscience, Columbia University, New York, NY, USA

${ }^{3}$ Molecular Medicine Program, Eunice Kennedy Shriver National Institute of Child Health and Human Development, National Institutes of Health, Bethesda, MD, USA

${ }^{4}$ Departamento de Fisiología Biofísica y Neurociencias, Centro de Investigación y de Estudios Avanzados del Instituto Politécnico Nacional, México City, México ${ }^{5}$ Institute of Zoology, University of Regensburg, Regensburg, Germany

Edited by:

Paolo Arosio, University of Brescia, Italy

\section{Reviewed by:}

Bing Zhou, Tsinghua University, China María-José Martínez-Sebastián, University of Valencia, Spain

\section{*Correspondence:}

Fanis Missirlis, Departamento de Fisiología Biofísica y Neurociencias, Centro de Investigación y de Estudios Avanzados del Instituto Politécnico Nacional, Avenida Instituto Politécnico Nacional 2508, Zacatenco, 07360 México City, District Federal, México e-mail: fanis@fisio.cinvestav.mx
Iron is required for organismal growth. Therefore, limiting iron availability may be a key part of the host's innate immune response to various pathogens, for example, in Drosophila infected with Zygomycetes. One way the host can transiently reduce iron bioavailability is by ferritin overexpression. To study the effects of neuronal-specific ferritin overexpression on survival and neurodegeneration we generated flies simultaneously over-expressing transgenes for both ferritin subunits in all neurons. We used two independent recombinant chromosomes bearing UAS-Fer1HCH, UAS-Fer2LCH transgenes and obtained qualitatively different levels of late-onset behavioral and lifespan declines. We subsequently discovered that one parental strain had been infected with a virulent form of the bacterial endosymbiont Wolbachia, causing widespread neuronal apoptosis and premature death. This phenotype was exacerbated by ferritin overexpression and was curable by antibiotic treatment. Neuronal ferritin overexpression in uninfected flies did not cause evident neurodegeneration but resulted in a late-onset behavioral decline, as previously reported for ferritin overexpression in glia. The results suggest that ferritin overexpression in the central nervous system of flies is tolerated well in young individuals with adverse manifestations appearing only late in life or under unrelated pathophysiological conditions.

Keywords: metal, symbiosis, rickettsia, popcorn, wMel, insect, immunity, aging

\section{INTRODUCTION}

Iron is essential for the growth of microorganisms and animals because it serves as a cofactor in many enzymes (Sheftel et al., 2012). Symbiotic relationships will therefore require the tuning of iron homeostasis between the organisms, whereas in parasitism, iron sequestration becomes an important factor in the antagonism between parasite and host (Cassat and Skaar, 2013). Drosophila melanogaster is used very widely to study basic biological questions and as a model of human disease (Rajan and Perrimon, 2013), but our knowledge of how iron homeostasis is maintained in Drosophila is still rather limited [reviewed in (Mandilaras et al., 2013; Tang and Zhou, 2013b)]. It is known that iron is normally stored in specialized intestinal cells expressing ferritin within their secretory system (Locke and Leung, 1984; Mehta et al., 2009). Ironloaded ferritin can be excreted to the intestinal lumen and is also found in the hemolymph (Locke and Leung, 1984). Disrupted ferritin function by mutation or RNA interference (RNAi) results in embryonic or first instar larval lethality (Missirlis et al., 2007; Li, 2010; Tang and Zhou, 2013a). Overexpression of ferritin also leads to excess iron sequestration and functional iron deficiency, which does not impede development to adulthood (Missirlis et al., 2007; Gutierrez et al., 2013; Tang and Zhou, 2013a). Neuronal ferritin overexpression was found to be beneficial when flies were fed with aluminum (Wu et al., 2012), whereas glial ferritin overexpression resulted in ferritin-iron inclusions in a subset of glia of the optic lobes and mild behavioral defects with a late-onset of appearance (Kosmidis et al., 2011). However, the effects of neuronal ferritin overexpression in otherwise wild type individuals have not been studied to date and were the first objective of this study.

Drosophila melanogaster laboratory cultures commonly host a symbiotic relationship with the $\alpha$-proteobacteria Wolbachia species, in which infected females show a reproductive advantage over non-infected, with no other overt fitness costs associated with the presence of the bacterium (Werren et al., 2008; Saridaki and Bourtzis, 2010). As an exception, a virulent Wolbachia strain causing degeneration and early death has been identified (Min and Benzer, 1997). Its name, popcorn (wMelPop) reflects the appearance of the bacteria visualized in situ with electron microscopy (EM) and is thought to result from its increased proliferation. The severity of $w$ MelPop-mediated phenotypes has been shown to vary with ambient temperature and the genetic background of the host (Reynolds et al., 2003). A whole-genome sequence comparison between variants of $w \mathrm{MelPop}$ and $w \mathrm{Mel}$ 
revealed only minor genetic changes between the pathogenic and symbiotic strains, providing no direct clues to help explain differences in virulence (Woolfit et al., 2013). Intriguingly, a small genomic region encoding 24 bacterial genes (out of a total of 1,111 annotated in the species) was either triplicated or absent in the pathogenic strains (Woolfit et al., 2013). Finally, two recent imaging studies documented in anatomical detail the presence of $w$ MelPop in the fly brain (Albertson et al., 2013; Strunov et al., 2013).

Previous results show that Wolbachia influences iron homeostasis in D. melanogaster and in the closely related species D. simulans (Brownlie et al., 2009; Kremer et al., 2009). When grown under iron-limiting conditions, D. melanogaster suffers reduced fecundity, but when infected with $w \mathrm{Mel}$, the flies laid significantly more eggs in four out of six experimental trials (Brownlie et al., 2009). The beneficial effect of $w \mathrm{Mel}$ was only seen under iron stress conditions, but the likely relevance of these experiments were supported by the finding that two out of the three natural populations tested were iron-deficient as collected in the wild. In a different paradigm, where Wolbachia is an obligate parasite of the wasp Asobara tabida, a study identified that both ferritin chains were induced in non-infected wasps (Kremer et al., 2009). To investigate whether Wolbachia affected ferritin iron in other insect species, iron accumulation in infected and uninfected $D$. simulans was determined. No difference was seen under normal dietary conditions, but when iron was supplemented in the diet infected individuals accumulated significantly less iron. Therefore, it appears that in a variety of insect species the presence of Wolbachia enhances tolerance to iron stress. We describe here the effect of neuronal ferritin overexpression in infected and uninfected D. melanogaster adults as they age and provide the first example where imposing an iron stress exacerbates the virulence of a pathogenic form of Wolbachia.

\section{MATERIALS AND METHODS FLY STOCKS}

The X-chromosome insertion of the pan-neuronal driver elavGal4 was obtained from the Bloomington Drosophila Stock Center (\#458). The construction of transgenic stocks UAS-Fer1HCH and UAS-Fer2LCH have been described in detail elsewhere (Missirlis et al., 2007). The wMelPop infection likely occurred from a balancer stock (not recorded) used to recover the UAS-Fer $1 \mathrm{HCH}$, UAS-Fer2LCH recombinant on the X-chromosome. Wolbachiainfected D. simulans flies were a kind gift from Kostas Bourtzis.

\section{MICROSCOPY}

Adult brains were prepared, cut, and stained as described (Kretzschmar et al., 1997). Ultrathin Epon plastic sections were post stained with $2 \%$ uranyl acetate, followed by Reynolds' lead citrate and stabilized for transmission electron microscopy (TEM) by carbon coating. Examination was done under an optical microscope or with a Zeiss EM10C/VR (Oberkochen, Germany) electron microscope at $40-80 \mathrm{kV}$.

\section{BEHAVIORAL ASSAYS}

Female adult flies collected $0-2$ days after eclosion were kept throughout their lifetime at $25^{\circ} \mathrm{C}$ in groups of 20 individuals per vial. For elicited-escape response tests flies were adapted for at least $20 \mathrm{~min}$ in an environment of $25^{\circ} \mathrm{C}$ and $70-80 \%$ humidity illuminated by red light. They were then placed in $14 \mathrm{~mL}$ polystyrene Falcon tubes individually and each fly was vortexed at the highest speed for 2-3 s and was tested twice in negative geotaxis and horizontal escape assays (Kosmidis et al., 2011). All flies were recorded for at least $1 \mathrm{~min}$ or until they reached their target of $10 \mathrm{~cm}$. A minimum 20 flies per genotype were tested for each experimental point and the data analyzed parametrically for statistical significance using the JMP and Prism software. Lifespan determinations used a minimum of 120 flies per genotype.

\section{PCR-DETECTION OF Wolbachia}

The presence of Wolbachia was determined by PCR using 16s rDNA Wolbachia-specific primers (Bourtzis et al., 1996). The primer sequences used were as follows: $5^{\prime}$-TTGTAGCCTGCTATGGTATAACT- $3^{\prime}$ and $5^{\prime}$-GAATAGGTATGATMTCATGT- $3^{\prime}$.

\section{RESULTS \\ PHENOTYPES OBSERVED BY FERRITIN OVEREXPRESSION IN ALL FLY NEURONS}

We used the pan-neuronal driver elav-Gal4 to overexpress both ferritin subunits simultaneously from recombinant chromosomes bearing transgene insertions of UAS-Fer1HCH and UASFer2LCH. As the X-chromosome was modified in one stock to carry the two UAS transgenes and in the other stock to carry the Gal4 insertion in the elav locus, we used only female flies in our experiments. Following eclosure, adult females were kept with their male siblings for 2 days to ensure successful mating and thereafter, female cohorts were kept under non-crowded conditions with biweekly transfers to fresh food. Flies bearing the transgenes alone were used as controls, but we note that UAS-Fer1HCH, UAS-Fer $2 \mathrm{LCH} /+$; +/+; +/+ females behaved differently depending on whether their mothers were infected with Wolbachia or not and this observation (reported further below) was unknown to us when experiments were originally planned and performed. In Figure 1 our controls were derived from uninfected white $(w)$ mutant mothers. We also tested the effect of neuronal overexpression of single subunits, as they have been previously shown to rescue degeneration associated with soluble $\beta$-amyloid overexpression (Rival et al., 2009). Once weekly during the lifespan of the flies, we monitored the time required for individual flies to climb $10 \mathrm{~cm}$ following a brief stimulus that elicited a negative geotactic response (Figure 1). The control flies used in this experiment showed a modest two-fold increase $(p<0.05)$ in the time required to perform this test during the sixth week of testing. Flies overexpressing either ferritin subunit alone behaved similar to controls through the fifth week, but presented significant performance impairment (a fourfold increase in time, $p<0.001$ ) when the aging-related decline initiated at week six. The flies overexpressing both ferritin subunits from the transgenes on the third chromosome showed a modest compromise at weeks 5 and 6 (threefold and sixfold increases; $p<0.0001$ ). The flies overexpressing both ferritin subunits from the transgenes on the $\mathrm{X}$ chromosome showed a robust mobility impediment one week earlier than controls (fourfold increase in time by week five, 


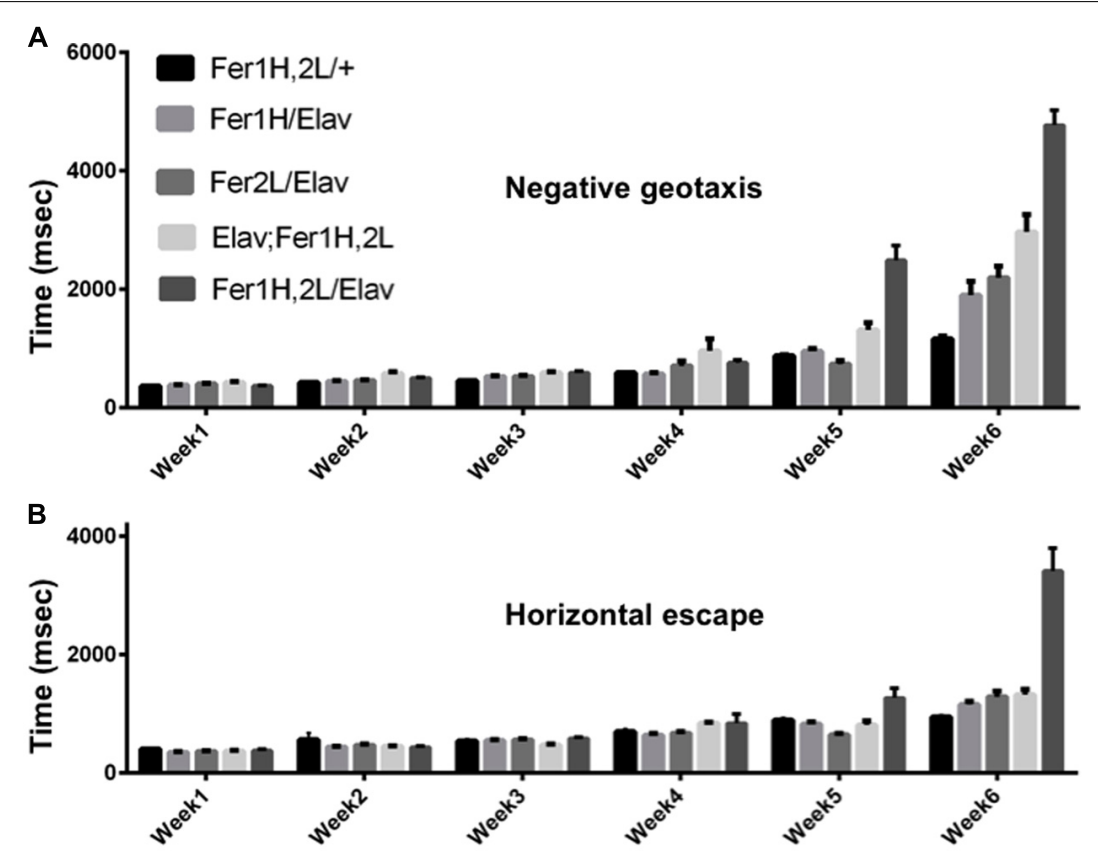

FIGURE 1 | Age dependent behavioral responses of flies overexpressing ferritin subunits pan-neuronally. Elicited escape response of flies was assessed on a weekly basis. The first bar on each graph corresponds to control (uninfected from Wolbachia) flies carrying the ferritin subunit transgenes without the elav-Gal4 driver. Bars 2 and 3 correspond to flies overexpressing Fer $1 \mathrm{HCH}$ and Fer $2 \mathrm{LCH}$, respectively. Bars 4 and 5 correspond to flies simultaneously overexpressing both Fer $1 \mathrm{HCH}$ and Fer2 $\mathrm{LCH}$ from recombinant transgenes on the third and $\mathrm{X}$ chromosomes, respectively.
(A) Flies expressing either the heavy or the light chain alone did not exhibit statistical differences from controls in their escape response until the fifth week and showed a moderate twofold increase in their response time on week 6 ( $p<0.05)$. In contrast, flies overexpressing both ferritin subunits showed a significant impairment at 5 and 6 weeks of age $(p<0.001)$.

(B) Only flies overexpressing both ferritin subunits from the $X$-chromosome were significantly compromised when responding in the horizontal direction in weeks 5 and $6(p<0.001)$. $p<0.001)$, with a dramatic tenfold increase in the time required to climb $10 \mathrm{~cm}$ by week six $(p<0.0001$; Figure 1A). Similar findings were found when testing horizontal escape responses, which presumably require less physical effort from individual flies (Figure 1B). During their sixth week post-eclosion, elavGal4/UAS-Fer1HCH, UAS-Fer $2 \mathrm{LCH}$; +/+; +/+ flies could hardly walk.

We then prepared plastic-embedded sections of adult fly heads to monitor the neuroanatomical integrity of the brains of 40-day old flies, at the transition between weeks 5 and 6. No apparent degeneration was evident in elav-Gal4/+; +/+; +/+ controls (Figure 2A), flies overexpressing the single ferritin subunits (Figures 2C,D) and those overexpressing ferritin from the third chromosomal recombinant (Figure 2E). In stark contrast, animals overexpressing ferritin from the $\mathrm{X}$-chromosome showed severe neuronal degeneration, evident throughout the brain, including the optic lobes (Figures 2B,F). Furthermore, lifespan determinations, clearly distinguished between ferritin overexpressing flies derived from the third and $\mathrm{X}$-chromosomal recombinants, with the latter presenting precipitous mortality during the sixth week of aging, 2 weeks earlier than controls (Figure 3A). Therefore, in addition to the significant difference in the onset of the mobility impediment between the two genotypes of flies overexpressing both ferritin subunits, brain pathology, and reduced lifespan were also associated only with the ferritin overexpression induced from the X-chromosomal transgenes.

\section{MATERNAL EFFECT ASSOCIATED WITH REDUCED LIFESPAN OF FERRITIN OVEREXPRESSING FLIES}

To investigate the potential cause of the differential shortened lifespan, neurodegeneration and early onset behavioral decline of the $\mathrm{X}$-chromosome driven ferritin overexpression, we tested whether there was a maternal effect associated with these phenotypes. We crossed homozygous female UAS-Fer $1 H C H$, UAS-Fer $2 L C H$ to male elav-Gal4 or to $w$ control flies and vice-versa. UAS-Fer $1 \mathrm{HCH}$, UAS-Fer2LCH/elav-Gal4 flies were therefore derived from UASFer1HCH, UAS-Fer2LCH or elav-Gal4 mothers, whereas UASFer $1 \mathrm{HCH}, \mathrm{UAS}$-Fer $2 \mathrm{LCH} /+$ control flies were derived from UASFer $1 H C H, U A S-F e r 2 L C H$, or $w$ mothers. The results clearly show that lifespan shortening was observed only in progeny of UASFer $1 \mathrm{HCH}, \mathrm{UAS}$-Fer2LCH mothers (Figure 3B). In agreement with this notion the decrease was also seen in control UAS-Fer1HCH, UAS-Fer $2 \mathrm{LCH} /+$ animals that do not overexpress ferritin since they do not carry the elav-Gal4 driver, but their transgene carrying chromosome is maternal in origin. Therefore, the behavioral, neurodegenerative, and reduced life span phenotypes are not associated with over-expression of the ferritin subunits, but rather with the maternal origin of the transgene-bearing chromosome.

\section{Wolbachia CLUSTERS DETECTED BY TEM AND PCR IN THE SEVERELY AFFECTED FERRITIN OVEREXPRESSING FLIES}

Transmission electron microscopy images from affected UASFer1HCH, UAS-Fer2LCH/elav-Gal4 flies revealed the presence of 


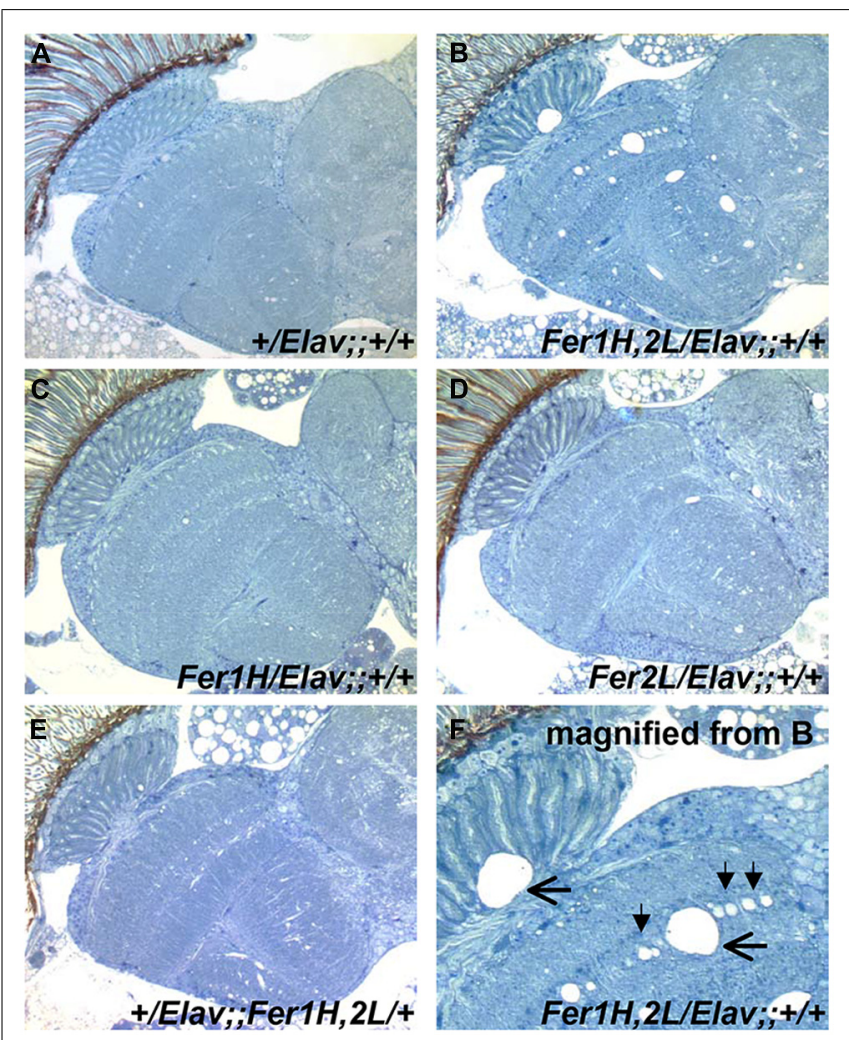

FIGURE 2 | Plastic embedded sections from 40-day old fly heads stained with toluidine blue. (A) Control flies show no signs of degeneration at this age (grown at $25^{\circ} \mathrm{C}$ ). (B) Flies overexpressing ferritin derived from $U A S-F e r 1 H C H, U A S-F e r 2 L C H ;+;+$ mothers crossed to Elav-Gal4; +; + fathers show extensive neurodegeneration. (C,D) Flies overexpressing single ferritin subunits show no signs of neurodegeneration. (E) Flies overexpressing ferritin from the recombinant insertions on the third Chromosome also show no apparent neurodegeneration. (F) Close-up of image shown in (B). Filled arrowheads denote the absence of single neuronal cell bodies lined up in the optic medulla. Horizontal arrows indicate areas of extensive neuronal damage.

bacterial infections, with the characteristic morphology attributed to $w$ MelPop (Figure 4A; Min and Benzer, 1997; Strunov et al., 2013). To verify the presence of Wolbachia independently, we performed genomic PCR using bacteria-specific primers on the UAS-Fer1HCH, UAS-Fer2LCH and elav-Gal4 parental lines and on UAS-Fer $1 \mathrm{HCH}$, UAS-Fer $2 \mathrm{LCH} /$ elav-Gal4 progeny derived from presumably infected and uninfected mothers (Figure 4B). We verified the presence of Wolbachia in mothers and progeny derived from the stock bearing the UAS-Fer1HCH, UAS-Fer2LCH recombinant on the $\mathrm{X}$-chromosome. We also assessed negative geotaxis on UAS-Fer1HCH, UAS-Fer2LCH/elav-Gal4 flies and in agreement with the life span experiment, we only observed a large time delay in infected individuals at 5 weeks of age (Figure 4C). Although the severe neurodegeneration that follows $w$ MelPop infection has been previously documented, it has been assumed that the bacterial infection leads to neuronal necrosis (Min and Benzer, 1997). Our TEM analysis demonstrated numerous pyknotic nuclei within CNS neurons (Figure 5), consistent with the characteristic nuclear changes that accompany programmed cell death. Treatment of the UAS-Fer1HCH, UAS-Fer2LCH stock with the antibiotic tetracycline for four consecutive generations eliminated the infection (Figure 6A). UAS-Fer1HCH, UASFer2LCH/elav-Gal4 flies derived from the tetracycline-treated UAS-Fer $1 H C H, U A S-F e r 2 L C H$ mothers retained their brain integrity at 40 days of age (Figures 6B,C) and, by qualitative observations, showed no signs of premature mobility decline and mortality.

\section{DISCUSSION}

\section{IRON AND Wolbachia SYMBIOSIS}

The maternally inherited bacterium Wolbachia infects arthropods and nematodes (Serbus et al., 2008). In filarial nematodes, Wolbachia is an obligate symbiont required for the successful reproduction of the worm (Bandi et al., 1999). The hypothesis that Wolbachia contributes metabolic provisioning to its hosts originated from whole genome sequencing projects showing that the entire heme biosynthesis pathway was present in Wolbachia but absent in Brugia malayi (Foster et al., 2005). This hypothesis was tested in D. melanogaster supporting the notion that the presence of Wolbachia assisted the flies under conditions of iron stress (Brownlie etal., 2009). Further support to the idea that iron is important in symbiotic relationships of Wolbachia and its hosts came from the finding that ferritin expression was induced in the absence of Wolbachia from A. tabida (Kremer et al., 2009). However, we still have no molecular understanding of the irondependent interactions between Wolbachia and its hosts. The same is true for interactions of Wolbachia with other metals (Wang et al., 2012) or with the host's antioxidant defense systems (Brennan et al., 2008).

Our study provided evidence that $w$ MelPop, or a $w$ MelPoplike strain, causes neuronal death, likely by inducing the apoptotic pathway in infected brains, with apoptosis seen also in the optic lobes, which generally were found to harbor significantly less bacteria (Albertson et al., 2013; Strunov et al., 2013). Ferritin overexpression, which likely results in depletion of readily bioavailable iron (Gutierrez et al., 2013), conferred no apparent protection to infected flies, but instead further decreased their shortened lifespan due to Wolbachia infection (Figure 3B). Our experiments did not discriminate between the possibility that ferritin overexpression benefited the pathogen, encouraging its propagation, or, alternatively, that ferritin overexpression on top of pathogen infection further deprived bioavailable brain iron. Our results point to a different outcome between $w$ MelPop and $w \mathrm{Mel}$ infections when responding to iron deficiency, with the former killing their host more effectively when the latter appear to provide an advantage to their host (Brownlie et al., 2009), although our observations call for further, differently controlled, experiments to settle this question. It will be of interest to investigate whether iron-related genes are included in the genomic region that appears to distinguish the pathogenic wMelPop strains from the more common endosymbiotic $w \mathrm{Mel}$ variants (Woolfit et al., 2013).

\section{IRON LIMITATION AND INSECT IMMUNITY}

The clearest evidence that iron availability can determine the outcome of an infection in Drosophila was provided by a study of 


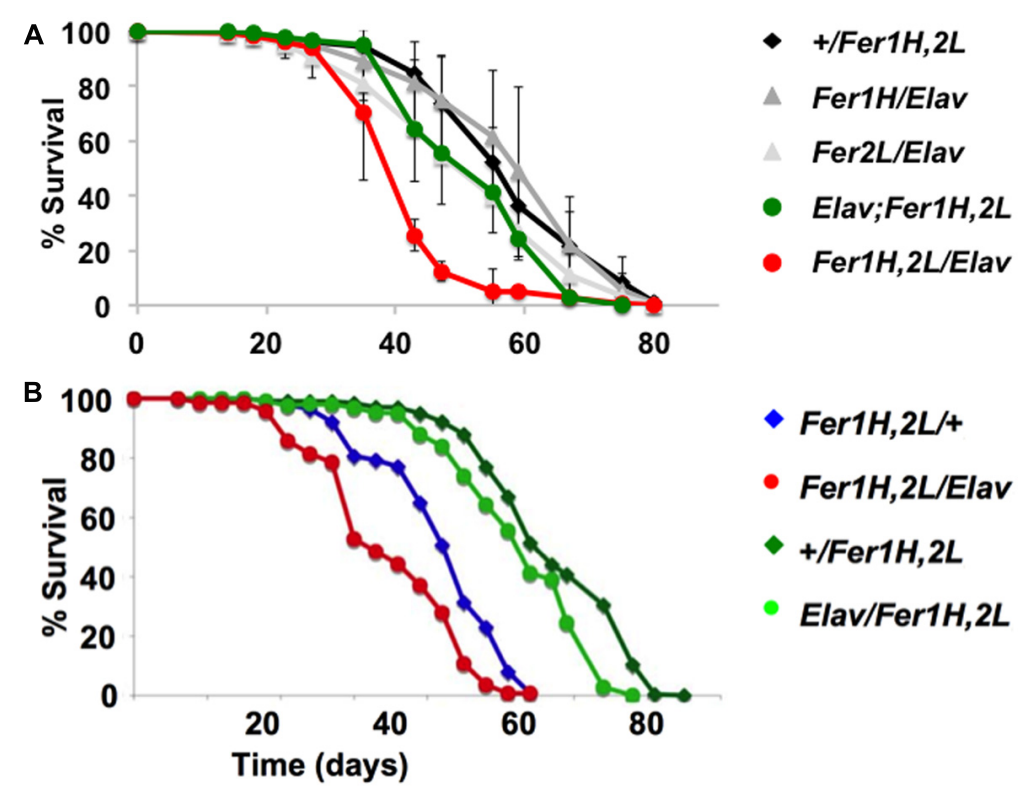

FIGURE 3 | Lifespan determinations of cohorts of female adult D. melanogaster. Day 0 marks eclosion, flies were kept with the opposite sex for the first five days, then they were separated on groups of 20 and transferred to fresh food regularly. (A) The same genotypes assayed in Figure 1 for behavior were used here. The experiments were performed three times independently (each time with a minimum cohort of 120 flies per genotype) and standard deviations are shown between the three experiments. Note the early onset of mortality in the flies overexpressing ferritin from the X-chromosomal recombinant. (B) A similar single experiment demonstrating that a maternal component determines the early onset mortality. Note that the same genotypes have markedly different lifespans, whose length is predicted by the mothers used for the crosses (see text for details). Overexpression of ferritin was never seen to have a beneficial effect on lifespan.

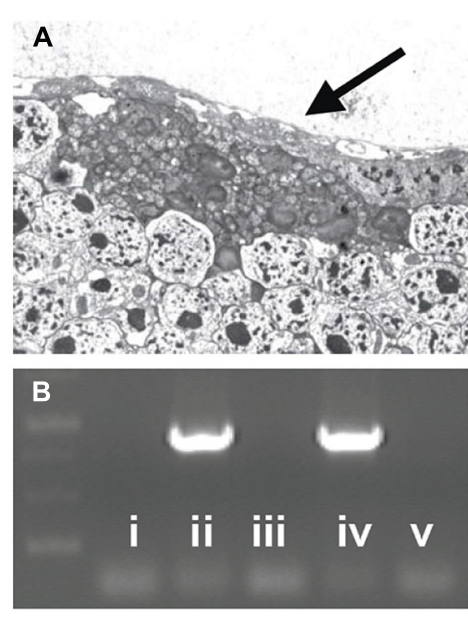

FIGURE 4 | (A) Bacteria accumulating amongst neurons and bearing the characteristic popcorn appearance of the wMelPop strain. (B) PCR detection of the Wolbachia ribosomal 16S gene in the following genotypes (i) no DNA, (ii) $U A S-F e r 1 H C H, U A S-F e r 2 L C H$ recombinant on $\mathrm{X}$, (iii) elav-Gal4 on $\mathrm{X}$, (iv) UAS-Fer1HCH, UAS-Fer2LCH/elav-Gal4

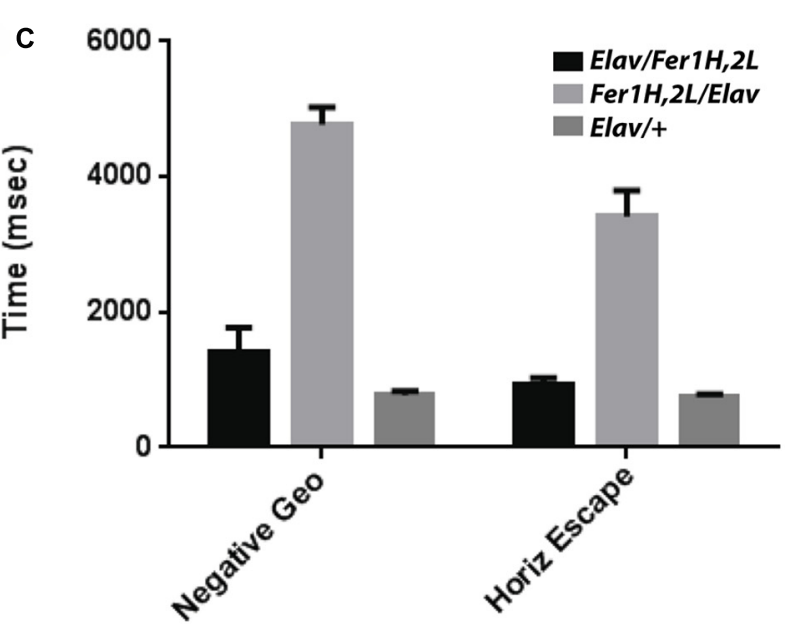

(mothers from ii), (v) Elav-Gal4/UAS-Fer1HCH, UAS-Fer2LCH (mothers from iii). (C) Behavioral responses of (v) - black bars - Elav-Gal4/ UAS-Fer1HCH, UAS-Fer2LCH, (iv) - light gray bars - UAS-Fer1HCH, UAS-Fer2LCH/elav-Gal4 and Elav-Gal4/+ control flies (dark gray bars) at 6 weeks of age. infection with the human fungal pathogen Zygomycetes (Chamilos etal., 2008). Provision of iron to the fungus resulted in more aggressive infections whereas treatment of flies with an iron chelator suppressed the infection. This outcome is consistent with the broadly recognized role of iron in infection: its availability is good for the pathogen and bad for the host
(Drakesmith and Prentice, 2012). Although very limited studies have been conducted investigating the role of iron during infections in Drosophila to date, the emerging picture appears to be complex and specific to each microbe studied. Hence, three qualitatively different interactions have been noted: (i) that iron limitation stops an infection (Chamilos et al., 2008), (ii) that 


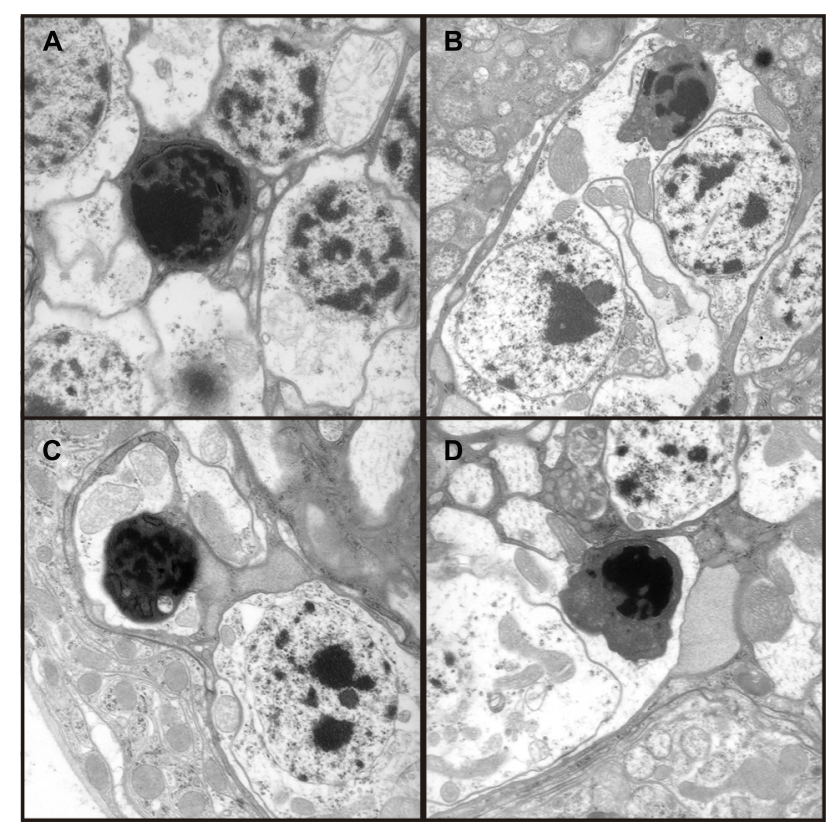

FIGURE 5 | (A-D) Four independent electron micrographs from Wolbachia infected flies showing characteristic apoptotic nuclei, suggesting neuronal death results from apoptosis.

iron limitation exacerbates the pathogenesis of an infection (this study), or (iii) that symbiotic infection becomes beneficial to the host under iron limitation (Brownlie et al., 2009). Clearly the role of iron in Drosophila immunity is an exciting open field for study.

\section{FERRITIN OVEREXPRESSION IN THE CENTRAL NERVOUS SYSTEM OF Drosophila}

We previously described the effect of ferritin overexpression in all Drosophila glia (Kosmidis et al., 2011) and in terms of behavior and lifespan, the major conclusions are very similar to these reported here for neurons: there seems to be no decrease in lifespan of flies overexpressing ferritin and there is only a small but readily quantifiable late-onset behavioral defect in negative geotactic responses. Although there is a detectable negative effect from ferritin overexpression at old age in wild type conditions, it has been clearly shown that ferritin overexpression can be protective under conditions of metal stress (Missirlis et al., 2007; Wu et al., 2012). Ferritin overexpression is thought to generate an iron limitation, but ferritin may be also able to sequester other metals with a significantly lower capacity (Gutierrez et al., 2013). One particularity of overexpressing ferritin in glia is that ironloaded ferritin accumulated in a subset of glia in the optic lobes forming inclusions (Kosmidis et al., 2011). No such accumulation was detected with ferritin overexpression in neurons. In addition, RNAi experiments against each ferritin subunit resulted in behavioral alterations when neurons were targeted and lethality when ferritin was suppressed in glia (Mandilaras and Missirlis, 2012; Tang and Zhou, 2013a). Our results suggest that ferritin overexpression, and iron chelators more generally (Soriano et al., 2013),

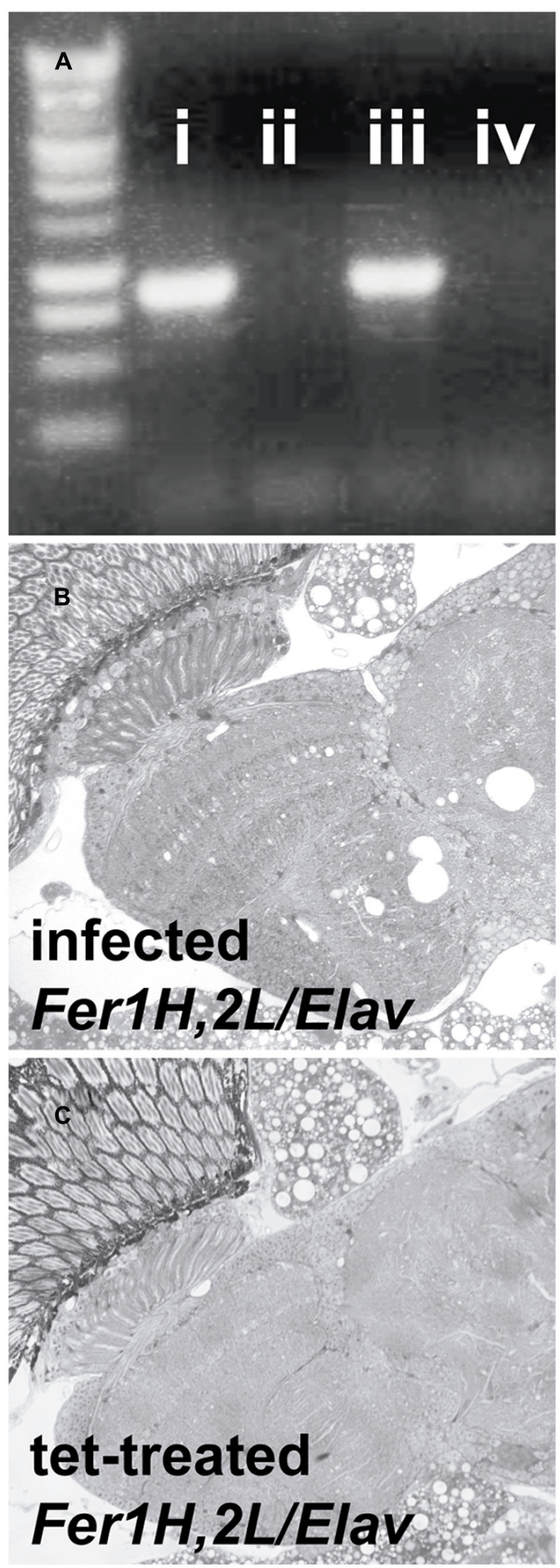

FIGURE 6 | Treatment with the antibiotic tetracycline cured the flies from the Wolbachia infection. (A) PCR detection of the Wolbachia ribosomal $16 \mathrm{~S}$ gene in (i) infected $D$. simulans (ii) tetracycline-treated $D$. simulans (iii) infected UAS-Fer1HCH, UAS-Fer2LCH on X (D. melanogaster) (iv) tetracycline-treated UAS-Fer1HCH, UAS-Fer2LCH on X (B) Plastic embedded sections of heads from infected (mothers from (iii) and (C) tetracycline-treated (mothers from iv) Elav/UAS-Fer1HCH, UAS-Fer2LCH adults at 40 days of age. 
should be considered as a therapeutic means of intervention, but a more general recommendation for iron chelation in otherwise healthy adults is not supported from the model organism D. melanogaster.

\section{AUTHOR CONTRIBUTIONS}

All authors designed the original set of experiments aiming to address the consequences of ferritin overexpression in neurons of wild type flies and participated in the data analysis. Fanis Missirlis generated the transgenic flies and measured the life spans. Stylianos Kosmidis performed the behavioral tests, the molecular biology to detect Wolbachia and the antibiotic treatments. Jose A. Botella and Stephan Schneuwly performed the electron microscopy analysis. Tracey A. Rouault and Efthimios M. C. Skoulakis had continuous input during each stage of the project, including during the writing of the manuscript, with Fanis Missirlis in a coordinating role.

\section{ACKNOWLEDGMENTS}

This research was supported by the Intramural Program of the Eunice Kennedy Shriver National Institute of Child Health and Human Development in the USA and the CONACYT project 179835 in Mexico. We thank Kostas Bourtzis for providing Wolbachia infected flies and advice for detecting the bacteria.

\section{REFERENCES}

Albertson, R., Tan, V., Leads, R. R., Reyes, M., Sullivan, W., and Casper-Lindley, C. (2013). Mapping Wolbachia distributions in the adult Drosophila brain. Cell. Microbiol. 15, 1527-1544. doi: 10.1111/cmi.12136

Bandi, C., McCall, J. W., Genchi, C., Corona, S., Venco, L., and Sacchi, L. (1999). Effects of tetracycline on the filarial worms Brugia pahangi and Dirofilaria immitis and their bacterial endosymbionts Wolbachia. Int. J. Parasitol. 29, 357-364.

Bourtzis, K., Nirgianaki, A., Markakis, G., and Savakis, C. (1996). Wolbachia infection and cytoplasmic incompatibility in Drosophila species. Genetics 144, 1063-1073.

Brennan, L. J., Keddie, B. A., Braig, H. R., and Harris, H. L. (2008). The endosymbiont Wolbachia pipientis induces the expression of host antioxidant proteins in an Aedes albopictus cell line. PLoS ONE 3:e2083. doi: 10.1371/journal.pone.00 02083

Brownlie, J. C., Cass, B. N., Riegler, M., Witsenburg, J. J., Iturbe-Ormaetxe, I., McGraw, E. A., et al. (2009). Evidence for metabolic provisioning by a common invertebrate endosymbiont, Wolbachia pipientis, during periods of nutritional stress. PLoS Pathog. 5:e1000368. doi: 10.1371/journal.ppat.1000368

Cassat, J. E., and Skaar, E. P. (2013). Iron in infection and immunity. Cell Host Microbe 13, 509-519. doi: 10.1016/j.chom.2013.04.010

Chamilos, G., Lewis, R. E., Hu, J., Xiao, L., Zal, T., Gilliet, M., et al. (2008). Drosophila melanogaster as a model host to dissect the immunopathogenesis of zygomycosis. Proc. Natl. Acad. Sci. U.S.A. 105, 9367-9372. doi: 10.1073/pnas.0709578105

Drakesmith, H., and Prentice, A. M. (2012). Hepcidin and the iron-infection axis. Science 338, 768-772. doi: 10.1126/science.1224577

Foster, J., Ganatra, M., Kamal, I., Ware, J., Makarova, K., Ivanova, N., et al. (2005). The Wolbachia genome of Brugia malayi: endosymbiont evolution within a human pathogenic nematode. PLoS Biol. 3:e121. doi: 10.1371/journal.pbio.0030121

Gutierrez, L., Zubow, K., Nield, J., Gambis, A., Mollereau, B., Lazaro, F. J., et al. (2013). Biophysical and genetic analysis of iron partitioning and ferritin function in Drosophila melanogaster. Metallomics 5, 997-1005. doi: 10.1039/c3mt0 $0118 \mathrm{k}$

Kosmidis, S., Botella, J. A., Mandilaras, K., Schneuwly, S., Skoulakis, E. M., Rouault, T. A., et al. (2011). Ferritin overexpression in Drosophila glia leads to iron deposition in the optic lobes and late-onset behavioral defects. Neurobiol. Dis. 43, 213-219. doi: 10.1016/j.nbd.2011.03.013
Kremer, N., Voronin, D., Charif, D., Mavingui, P., Mollereau, B., and Vavre, F. (2009). Wolbachia interferes with ferritin expression and iron metabolism in insects. PLoS Pathog. 5:e1000630. doi: 10.1371/journal.ppat.1000630

Kretzschmar, D., Hasan, G., Sharma, S., Heisenberg, M., and Benzer, S. (1997). The swiss cheese mutant causes glial hyperwrapping and brain degeneration in Drosophila. J. Neurosci. 17, 7425-7432.

Li, S. (2010). Identification of iron-loaded ferritin as an essential mitogen for cell proliferation and postembryonic development in Drosophila. Cell Res. 20, 11481157. doi: $10.1038 / \mathrm{cr} .2010 .102$

Locke, M., and Leung, H. (1984). The induction and distribution of an insect ferritin-a new function for the endoplasmic reticulum. Tissue Cell 16, 739-766. doi: 10.1016/0040-8166(84)90007-7

Mandilaras, K., and Missirlis, F. (2012). Genes for iron metabolism influence circadian rhythms in Drosophila melanogaster. Metallomics 4, 928-936. doi: $10.1039 / \mathrm{c} 2 \mathrm{mt} 20065 \mathrm{a}$

Mandilaras, K., Pathmanathan, T., and Missirlis, F. (2013). Iron absorption in Drosophila melanogaster. Nutrients 5, 1622-1647. doi: 10.3390/nu5051622

Mehta, A., Deshpande, A., Bettedi, L., and Missirlis, F. (2009). Ferritin accumulation under iron scarcity in Drosophila iron cells. Biochimie 91, 1331-1334. doi: 10.1016/j.biochi.2009.05.003

Min, K. T., and Benzer, S. (1997). Wolbachia, normally a symbiont of Drosophila, can be virulent, causing degeneration and early death. Proc. Natl. Acad. Sci. U.S.A. 94, 10792-10796.

Missirlis, F., Kosmidis, S., Brody, T., Mavrakis, M., Holmberg, S., Odenwald, W. F., et al. (2007). Homeostatic mechanisms for iron storage revealed by genetic manipulations and live imaging of Drosophila ferritin. Genetics 177, 89-100. doi: 10.1534/genetics.107.075150

Rajan, A., and Perrimon, N. (2013). Of flies and men: insights on organismal metabolism from fruit flies. BMC Biol. 11:38. doi: 10.1186/1741-7007-11-38

Reynolds, K. T., Thomson, L. J., and Hoffmann, A. A. (2003). The effects of host age, host nuclear background and temperature on phenotypic effects of the virulent Wolbachia strain popcorn in Drosophila melanogaster. Genetics 164, 1027-34.

Rival, T., Page, R. M., Chandraratna, D. S., Sendall, T. J., Ryder, E., Liu, B., et al. (2009). Fenton chemistry and oxidative stress mediate the toxicity of the betaamyloid peptide in a Drosophila model of Alzheimer's disease. Eur. J. Neurosci. 29, 1335-1347. doi: 10.1111/j.1460-9568.2009.06701.x

Saridaki, A., and Bourtzis, K. (2010). Wolbachia: more than just a bug in insects genitals. Curr. Opin. Microbiol. 13, 67-72. doi: 10.1016/j.mib.2009.11.005

Serbus, L. R., Casper-Lindley, C., Landmann, F., and Sullivan, W. (2008). The genetics and cell biology of Wolbachia-host interactions. Annu. Rev. Genet. 42, 683-707. doi: 10.1146/annurev.genet.41.110306.130354

Sheftel, A. D., Mason, A. B., and Ponka, P. (2012). The long history of iron in the Universe and in health and disease. Biochim. Biophys. Acta 1820, 161-187. doi: 10.1016/j.bbagen.2011.08.002

Soriano, S., Llorens, J. V., Blanco-Sobero, L., Gutierrez, L., Calap-Quintana, P., Morales, M. P., et al. (2013). Deferiprone and idebenone rescue frataxin depletion phenotypes in a Drosophila model of Friedreich's ataxia. Gene 521, 274-281. doi: 10.1016/j.gene.2013.02.049

Strunov, A., Kiseleva, E., and Gottlieb, Y. (2013). Spatial and temporal distribution of pathogenic Wolbachia strain wMelPop in Drosophila melanogaster central nervous system under different temperature conditions. J. Invertebr. Pathol. 114, 22-30. doi: 10.1016/j.jip.2013.05.001

Tang, X., and Zhou, B. (2013a). Ferritin is the key to dietary iron absorption and tissue iron detoxification in Drosophila melanogaster. FASEB J. 27, 288-298. doi: 10.1096/fj.12-213595

Tang, X. N., and Zhou, B. (2013b). Iron homeostasis in insects: insights from Drosophila studies. IUBMB Life 65, 863-872. doi: 10.1002/iub.1211

Wang, L., Zhou, C., He, Z., Wang, Z. G., Wang, J. L., and Wang, Y. F. (2012). Wolbachia infection decreased the resistance of Drosophila to lead. PLoS ONE 7:e32643. doi: 10.1371/journal.pone.0032643

Werren, J. H., Baldo, L., and Clark, M. E. (2008). Wolbachia: master manipulators of invertebrate biology. Nat. Rev. Microbiol. 6, 741-751. doi: 10.1038/nrmicr o1969

Woolfit, M., Iturbe-Ormaetxe, I., Brownlie, J. C., Walker, T., Riegler, M., Seleznev, A., et al. (2013). Genomic evolution of the pathogenic Wolbachia strain, wMelPop. Genome Biol. Evol. 5, 2189-2204. doi: 10.1093/gbe/evt169

Wu, Z., Du, Y., Xue, H., Wu, Y., and Zhou, B. (2012). Aluminum induces neurodegeneration and its toxicity arises from increased iron accumulation and reactive 
oxygen species (ROS) production. Neurobiol. Aging 33, 199.e1-199.e12. doi: 10.1016/j.neurobiolaging.2010.06.018

Conflict of Interest Statement: The authors declare that the research was conducted in the absence of any commercial or financial relationships that could be construed as a potential conflict of interest.

Received: 21 February 2014; paper pending published: 10 March 2014; accepted: 20 March 2014; published online: 04 April 2014.

Citation: Kosmidis S, Missirlis F, Botella JA, Schneuwly S, Rouault TA and Skoulakis EMC (2014) Behavioral decline and premature lethality upon pan-neuronal ferritin overexpression in Drosophila infected with a virulent form of Wolbachia. Front. Pharmacol. 5:66. doi: 10.3389/fphar.2014.00066

This article was submitted to Drug Metabolism and Transport, a section of the journal Frontiers in Pharmacology.

Copyright (C) 2014 Kosmidis, Missirlis, Botella, Schneuwly, Rouault and Skoulakis. This is an open-access article distributed under the terms of the Creative Commons Attribution License (CC BY). The use, distribution or reproduction in other forums is permitted, provided the original author(s) or licensor are credited and that the original publication in this journal is cited, in accordance with accepted academic practice. No use, distribution or reproduction is permitted which does not comply with these terms. 